\title{
Some Unexpected Difficulties in Microscope Operation in Microgravity
}

\author{
Donald Pettit ${ }^{1}$
}

1. NASA, Johnson Space Center, Houston, TX

The International Space Station (ISS) is a research laboratory in low earth orbit where the magnitude of gravitational forces are reduced by a factor of one million. Changing any other earthly parameter by this magnitude rapidly takes one into an experimental frontier, and this orbital environment is no exception. Many facilities typical of ground-based laboratories are onboard ISS: furnaces, centrifuges, freezers, incubators, plant growth and combustion chambers, with supplied resources of vacuum, inert gas, oxygen, liquid and forced air cooling, 28 and 120 volt DC power, and near-continuous real-time communication of data, voice and high definition video transmitted over distances approaching 50,000 miles (roundtrip to geostationary orbit). Microscope facilities include many state-of-the-art imaging techniques: transmission, reflection, brightfield, darkfield, epi illumination, phase contrast, differential interference contrast, fluorescent, confocal, polarization, and student educational instruments [1]. During the operation of these microscopes in low earth orbit, some unexpected difficulties unrelated to the undergoing research but directly resulting from their operation in microgravity can delay the progress of an experiment. Difficulties can stem from errant fluid behavior, residual gravity gradients, cosmic rays, and safety of flight.

Subtle forces stemming from surface tension, liquid-solid contact angle, and static electric charge dominate fluid behavior in microgravity. These can conspire to give non-intuitive behaviors [2] resulting in possible operational delays or equipment maintenance. The precise placement of immersion oil on a slide using a pipette can be challenging (Fig. 1 left). Filling a sample chamber with bubble free liquid requires significant on-orbit practice. Flow induced charging of liquids, a small charge developed when a dielectric fluid (such as immersion oil) is forced through a small insulated capillary (such as a Teflon pipette), can result in subtle charge forces making the liquid misbehave [3]. These subtle forces under microgravity can interfere with the sample placement within the optical path (bubbles) or result in the fouling of optical surfaces (Fig. 1 center). The time necessary to learn the handling skills or to keep the instrument in operating order can cause delays in experimental progress.

The magnitude of residual acceleration on ISS is near $1.2 \mathrm{E}-6 \mathrm{~g}$, nominally referred to as microgravity, where $\mathrm{g}$ is the acceleration due to gravity on the surface of Earth. At this level of residual acceleration, sample motion is possible [4,5]. The direction of this residual acceleration in relation to the orientation of the experimental sample can cause unexpected fluid-particle-bubble motion within a sample chamber. Such motion might cause the intended subject to settle out of suspension or migrate outside the optical field of view over a period of a few hours.

The flux of cosmic rays in low earth orbit causes camera CCD or CMOS detector arrays to degrade after periods of about one year. They produce images strewn with hot pixel "snow" that can compromise their scientific usefulness (Fig 1. right). By design, some instrument cameras were never meant to be replaced, and after years in orbit, can suffer significant image degradation. A maintenance plan including periodic camera replacement should be considered.

When subjected to microgravity while living in a sealed thin shell surrounded by infinite vacuum, flight 
crew safety becomes paramount. Standard means of conducting research on ISS may require seemingly prohibitive constraints when compared to similar ground-based research. Other than water (recovered from urine,) there are no cleaning solvents available on ISS due to their detrimental effects on the regenerative life support systems. The lack of solvents complicates cleaning of optics and other delicate surfaces especially from unintended fluid migration (Fig. 1 center). Microgravity promotes the possibility of inhalation or eye damage from small free floating objects with possible serious consequences. On ISS, the handling of small parts (screws and nuts) or shards created from accidental breakage of fragile glass components (cover slips and slides) presents a significant crew hazard. Laborious, time-consuming practices are often required for what would normally be a trivial operation on Earth. Sometimes a compromise must be made between the safety requirements needed for the best scientific practices versus non-optimum materials, substituted to ease the handling requirements, such as plastic cover slips and slides.

[1] https://iss-science.jsc.nasa.gov/investigation_detail.cfm?investigationsid=541

[2] Pettit, D, "Exploring the Frontier: Science of Opportunity on the International Space Station", Proc. Am. Philo. Soc. vol. (153), No. 4, Dec. 2009, pp. 381-402.

[3] Pettit, D, "Flow Induced Charging of Liquids in Reduced Gravity", Eng. Const. \& Op. in Space, Space 96, S Johnson, Ed., ASCE Pub. Vol. I (1996), pp.545-551.

[4] Alexander, I, and Lundquist, C, "Residual Motions Caused by Micro-Gravitational Accelerations," Jour. Astro. Sci., Vol (35), no. 2, 1987, pp 193-211.

[5] Delombard, R, Kelly, E, Hrovat, K, Nelson, E, Pettit, D, "Motion of Air Bubbles in Water Subjected to Microgravity Accelerations", 43 ${ }^{\text {rd }}$ AIAA, Reno, Jan. (2005), AIAA-2005-0722.
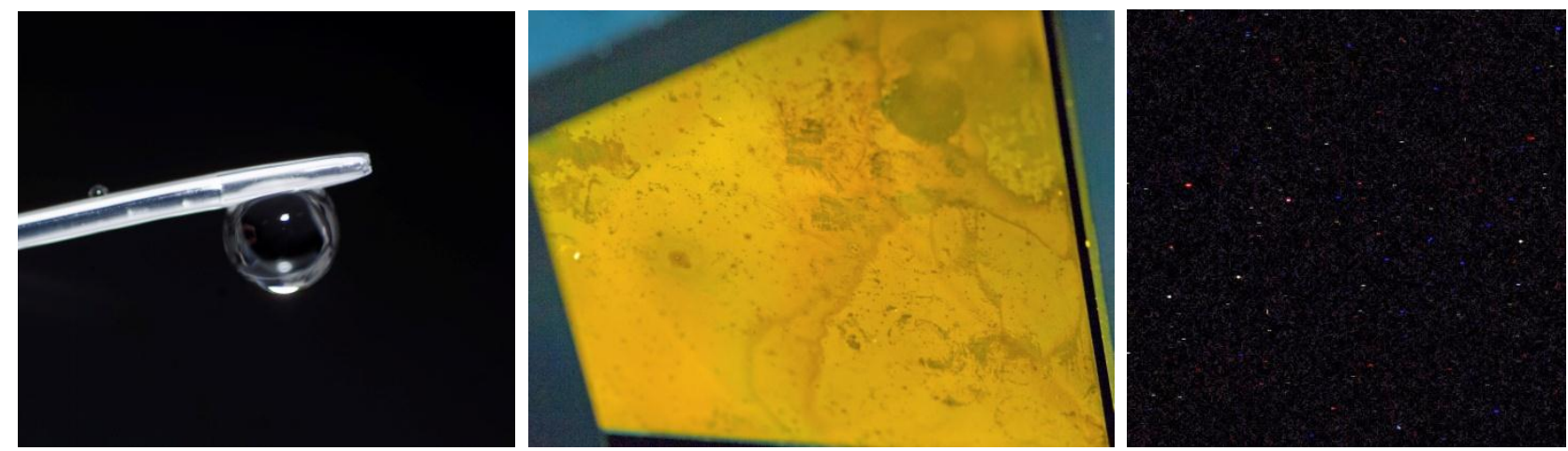

Figure 1. Examples of unexpected experimental difficulties on ISS: pipette tip (1.5mm diameter) showing migration of a water drop from the tip to the side during fluid operations on Exp. 6 (left) complicating its precise placement; front surfaced gold mirror $(100 \mathrm{~mm}$ wide) inadvertently contaminated with silicone oil that migrated from the experimental stage during Exp. 30 requiring about two hours of crew time with a three week schedule delay to clean (center); and a 450 by 450 pixel enlargement from a camera C-MOS detector dark frame image showing red-green-blue-white hot pixels from one year of cosmic ray damage during Exp. 42 (right). 